\title{
PASO SUPERIOR DEL ENLACE DE FONTIÑAS EN LA NUEVA RED PERIFÉRICA DE SANTIAGO DE COMPOSTELA. RETORNO A STONEHENGE
}

\author{
(THE OVERPASS OF THE FONTIÑAS INTERCHANGE ON THE NEW RING ROAD NETWORK OF \\ SANTIAGO DE COMPOSTELA. RETURN TO STONEHENGE)
}

Fernando da Cunha Rivas, Ingeniero de Caminos

ESPAÑA

Fecha de recepción: 23-VI-95

$510-16$

\section{RESUMEN}

Dada la peculiar geometria del enlace, obligada por la topografia y el estricto desarrollo de la glorieta y rampas de acceso, con radios muy pequeños, se comenta el proceso de su diseño e intuición del trabajo estructural, así como una somera definición de su cálculo y detalles constructivos, constituyendo una estructura singular en hormigón, dotada de gran esbeltez y aspecto global agradable.

Se describen las distintas fases y zonas de pretensado dejando grandes ventanas de tesado, e incluso cordones pasantes con anclajes ciegos, que configuran en su conjunto un "cosido" de toda la estructura, como puede observarse en las figuras y fotos que se acompañan.

\section{SUMMARY}

Given the peculiar geometry of the interchange, conditioned by the topography and the strict development of the roundabouts and underpass approaches, with very small radii, the article comments on the process of its design and the intuition of the structural work, and gives a brief definition of its calculation and construction details which make an outstanding concrete structure with great slenderness and an overall pleasant aspect.

A description is given of different prestressing stages and areas which leave great tensioning windows and even auxiliary chords with blind anchors, which all together form a "union" of the whole structure, as can be seen in the figures and photos.

\section{Introducción}

La nueva ronda de Santiago de Compostela discurre por un estrecho pasillo, relacionado con el núcleo urbano en enlaces perimetrales.

El enlace de Fontiñas se hace emblemático por el conjunto de la obra, por la visibilidad desde su entorno y por su forma peculiar.
La geometría de la obra viene obligada por razones topográficas y de gestión de terrenos, ya que la ronda en esa zona va tangente al FFCC Santiago - La Coruña, lo que configura una glorieta elevada, que desemboca en sendos ramales de entrada y salida.

El desarrollo de la glorieta y de las rampas, permite salvar la diferencia de cota entre las vías transversales a la ronda y el núcleo de ésta. 


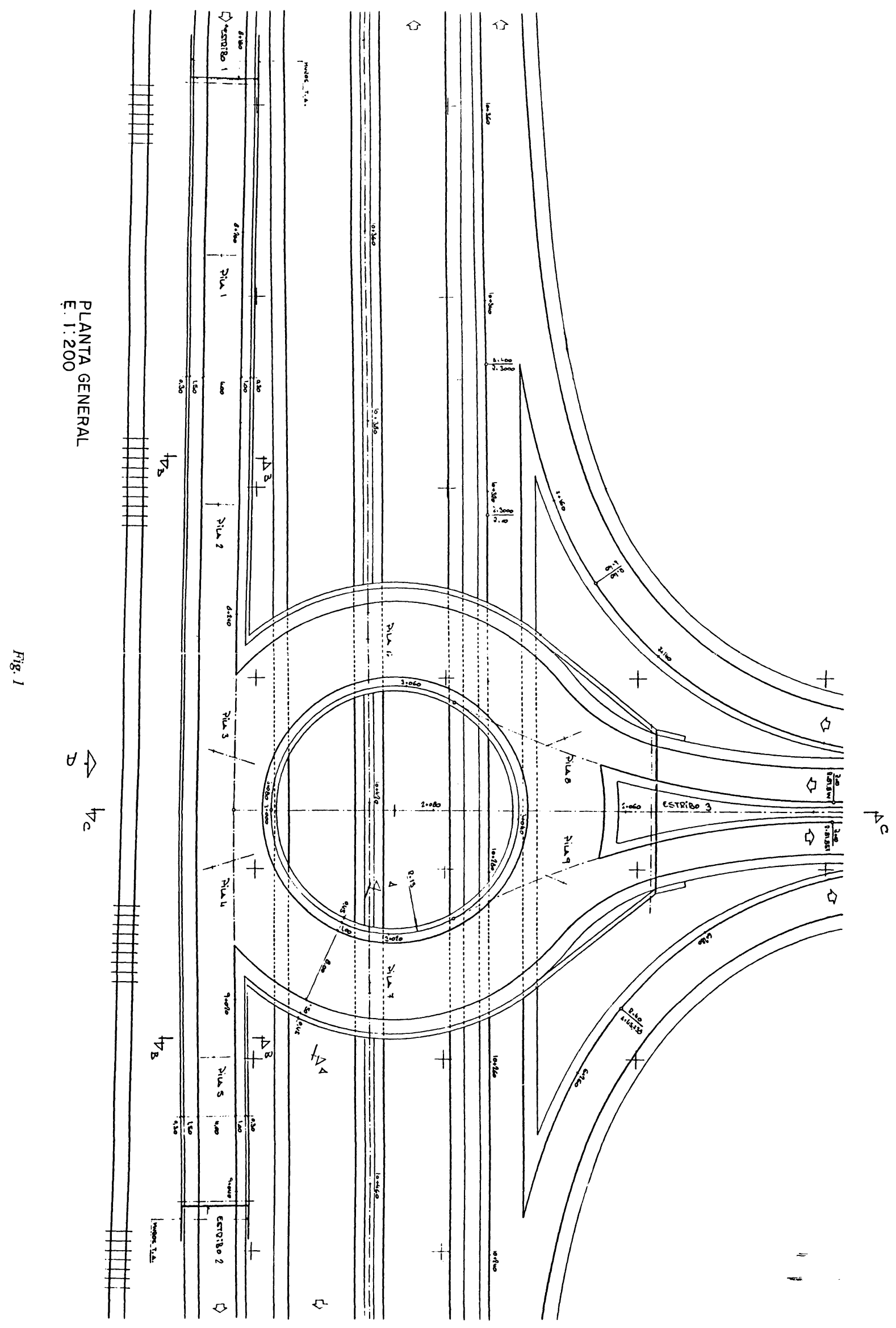




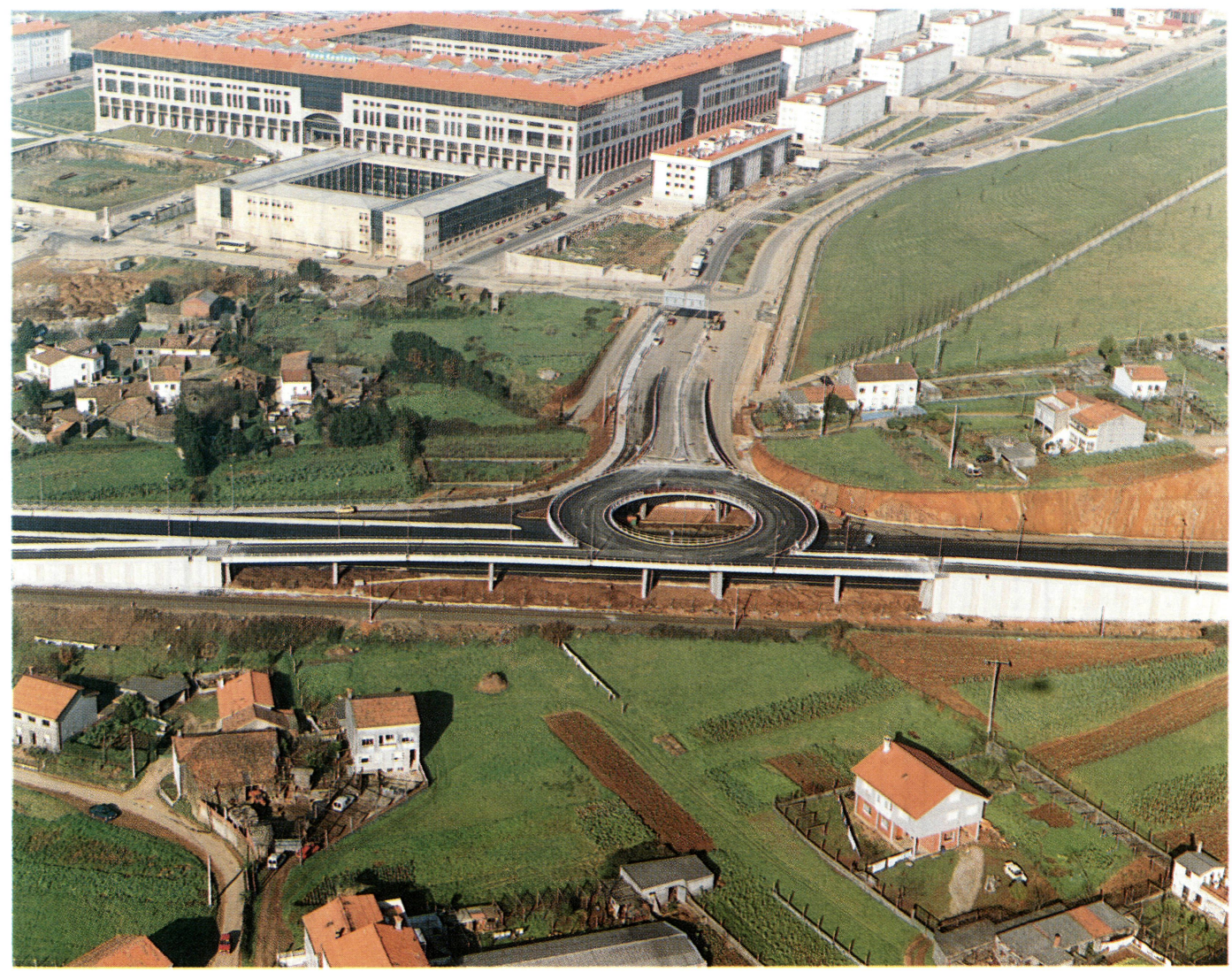

Vista panorámica.

Se genera así un paso superior, cuya planta tiene forma de "coca" (Fig. 1), es decir, se organiza mediante una corona circular y una banda recta que se intersectan en una pequeña zona, de unos $12 \mathrm{~m}$ de longitud.

El tramo recto tiene en total $117 \mathrm{~m}$ de longitud (Fig. 2), divididos en 6 vanos, de $18+26+26+12$ (unión con la corona circular) $+20+15$, contados desde el estribo 1 hasta el estribo 2 , con un ancho de $7,10 \mathrm{~m}$.

La corona circular, con $18,25 \mathrm{~m}$ de radio en el eje y con $11,40 \mathrm{~m}$ de ancho, se organiza en un vano de $10,44 \mathrm{~m}$, común con la zona recta, y sendos vanos de $20,88+26,50$ $\mathrm{m}$, medidos en el eje.

La obra se remata con una zona trapecial, de $17 \mathrm{~m}$ de ancho mínimo, en el estribo 3, que une la corona circular a la vía transversal (Fig. 3).

Toda la obra es monolítica, construida en hormigón pretensado por tres fases sucesivas, con un canto de $1 \mathrm{~m}$.

En la zona recta, la sección transversal es una artesa, con un fondo de $2 \mathrm{~m}$ y sendos voladizos de $1,65 \mathrm{~m}$ de luz, con espesor constante de $25 \mathrm{~cm}$ (Fig. 4).
En la corona circular, la sección, también en forma de artesa, tiene un fondo de $3,20 \mathrm{~m}$ y voladizos de $2,00 \mathrm{~m}$ (Fig. 5).

La altura de pilas y estribos es del orden de 6 m (Figs. 6 y 7).

El terreno en la zona es rocoso, con un recubrimiento no competente que oscila entre 4 y $12 \mathrm{~m}$ en las zonas

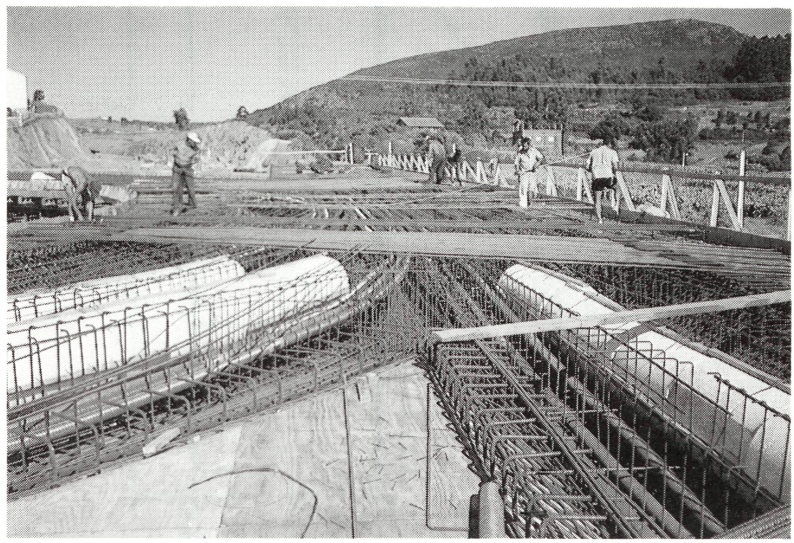

Detalle de la armadura y aligeramiento. 


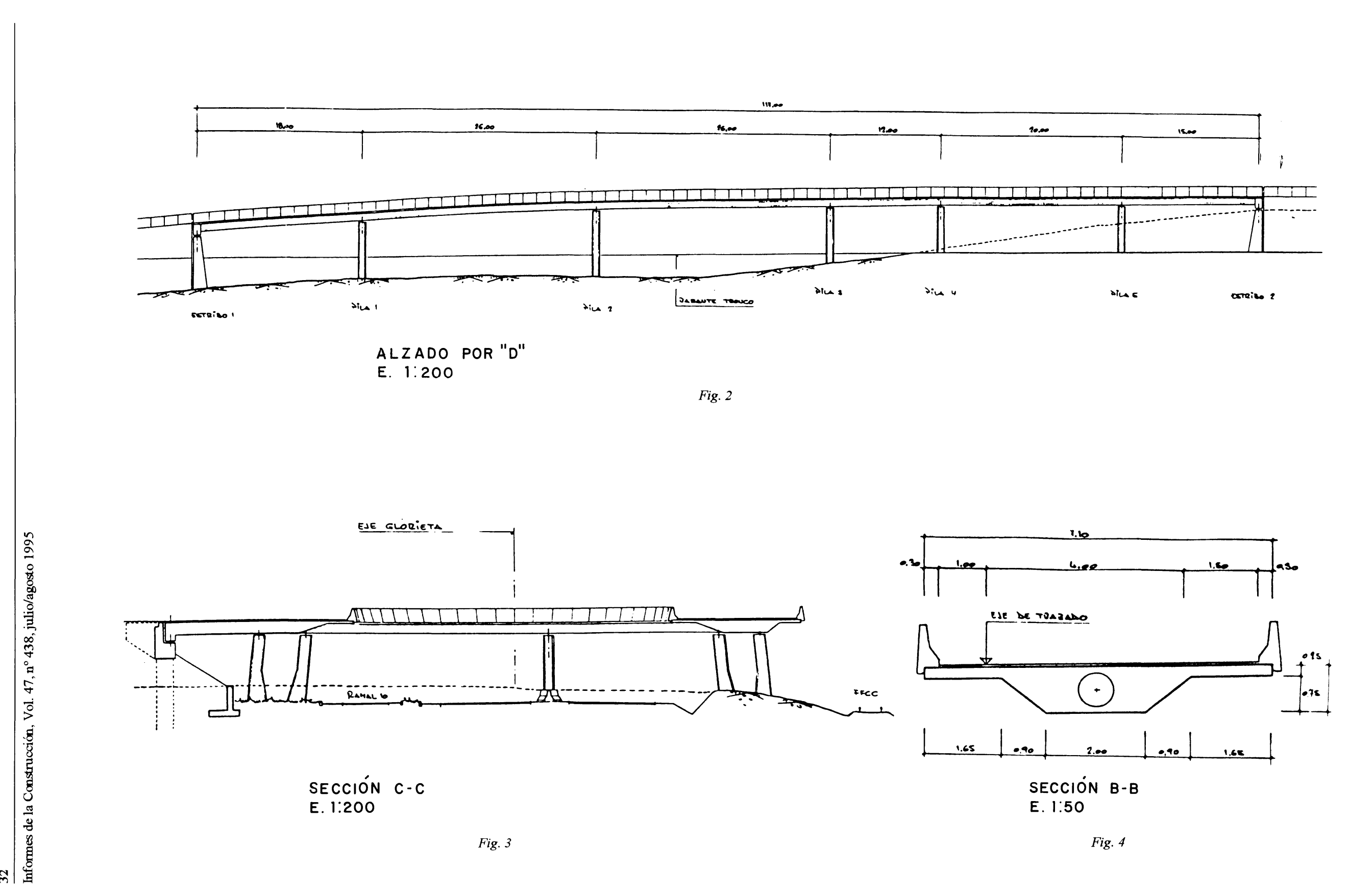




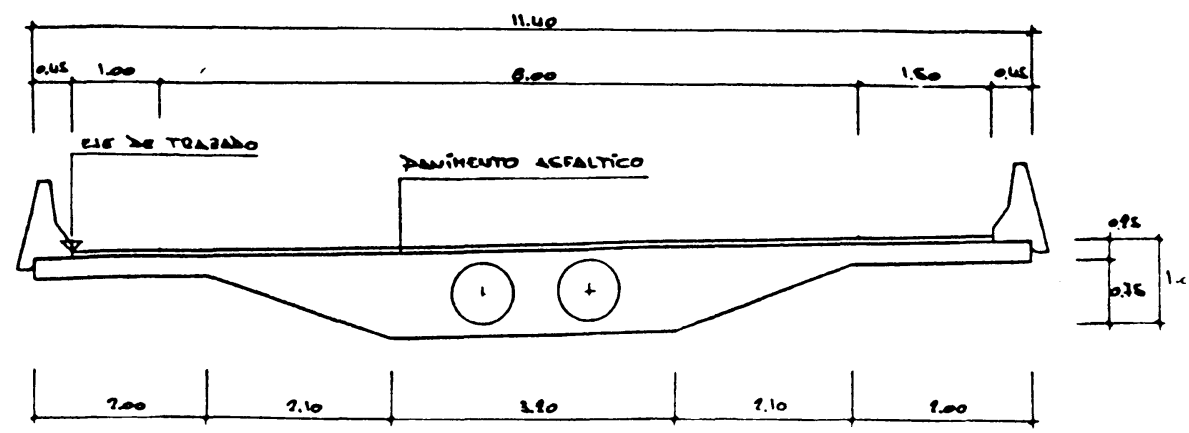

SECCION A-A

Fig. 5

E. 1:50

profundas, y menos de $2 \mathrm{~m}$ en las someras. La roca se presenta con un morro elevado que abarca a todos los apoyos, salvo los estribos y la pila 1 .

Suponemos que esta forma tan peculiar de la estructura no es una primicia, dado lo complejo de la geometría de los enlaces de la carretera, pero nosotros no fuimos capaces de encontrar los eventuales antecedentes, por lo que acometimos la obra tratando de extraer de nuestras anteriores experiencias, en puentes curvos y construidos por fases sucesivas, las instrucciones necesarias para su diseño.

\section{Diseño}

Los condicionantes iniciales de la obra fueron, por una parte, que el material a emplear era hormigón, por coherencia con el resto de la obra, y que sólo deberían existir juntas en los tres estribos.

Se pretendía que la obra tuviese un tratamiento formal no rutinario, cuestión de la que era difícil evadirse, a la vista de su curiosa planta.

Para buscar un aspectoglobal agradable, se trató de limitar el canto, que se fijó en $1 \mathrm{~m}$, dado que existía una luz de 26,50 metros en curva muy pronunciada, imposible de reducir, ya que es la distancia entre la mediana y los bordes de arcén. Con este canto y esa luz, el tablero debía se pretensado, lo cual, en un puente tan curvo, significa desperdiciar el $35 \%$ en pérdidas por rozamiento.

Se trató de evitar el efecto ciempiés, dotando de pilas de fuste único ataluzadas al tablero en las rampas rectas,y tratando de que las pilas en la zona curva, que creíamos debían proporcionar doble apoyo, evocasen un elemento único, armonizado con la planta, para lo cual nos ayudó mucho la visión de las ruinas de Stonehenge.

Nos pareció que una pequeña inclinación del $10 \%$ en las pilas de la zona curva podía ser relacionada intuitivamente con un descentramiento de la reacción en la zapata, para absorber la excentricidad producida por los fuertes torsores del tablero, sin duda recordando los afanes didácticos de Maillart.

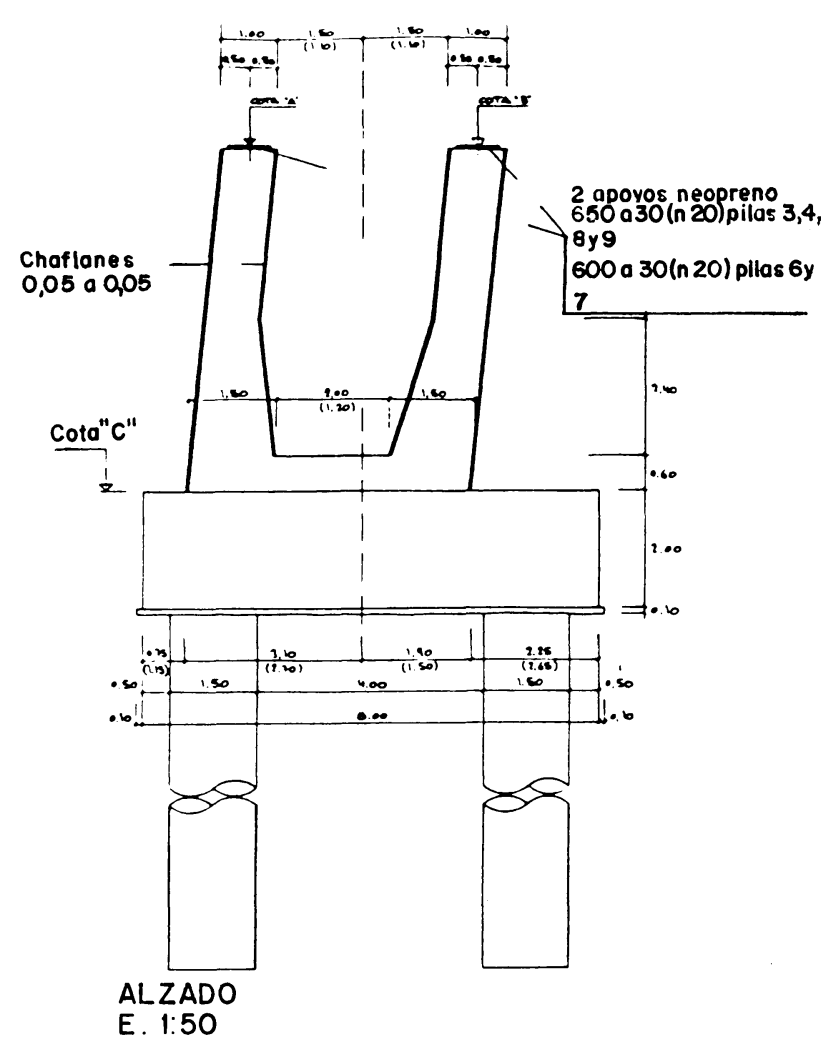

Fig. 6

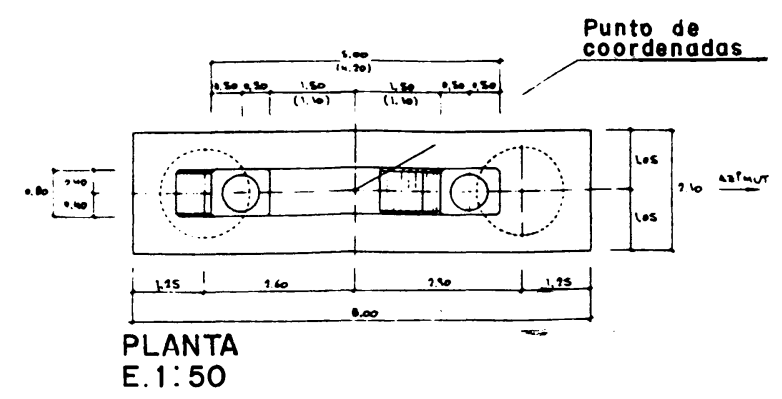

Fig. 7 
Finalmente, y como consecuencia de las decisiones tomadas sobre las pilas, los fondos de artesa debían ser poco anchos, para evitar la excesiva separación de las pilas dobles, lo que configuraba unos costeros muy inclinados, agradables a la vista e igualmente a la rigidez de torsión.

Para facilitar el encuentro entre las zonas recta y curva se dispusieron voladizos de canto constante, e igual a $25 \mathrm{~cm}$, en ambas secciones.

\section{Intuición del trabajo estructural}

El proyecto de esta obra tenía un plazo muy limitado, circunstancia no por repetida menos molesta, lo que obligaba a establecer un diseño concreto y desarrollarlo, sin tiempo para un proceso de "feed-back".

Intuímos un trabajo estructural basado en un funcionamiento lo menos encadenado posible entre el puente recto y el curvo, para lo cual se dispuso de una zona de neutralización común a ambos.

Al diseñar pilas de doble apoyo en la zona común, tratamos de limitar la influencia de las deformaciones torsoras en la conducta del tablero recto. Así, éste, debería comportarse como dos trozos rectos, unidos por un estribo formado por una losa elástica, más rígida que ellos, por tener menos luz.

Debido a lo diminuto del radio y lo limitado del canto, tratamos de aumentar la componente de respuesta a flexión transversal, en detrimento de la respuesta a torsor del tablero curvo, dotando a las pilas de la corona circular de doble apoyo. La zona trapecial debería ofrecer, por razones similares a las expuestas respecto a la "zona de neutralización", un empotramiento elástico que promoviese la primera de las respuestas, alejando así el fantasma de la no validez de la sección a cortante - torsión.

Dado que contábamos con un morro de roca somero bajo las pilas de la corona, estábamos en condiciones de limitar los giros de torsión sin apuros.

\section{Detalles constructivos}

Una vez dibujada la estructura, (a lápiz por supuesto) procedimos a pergeñar su proceso constructivo. Dada la enorme superficie de la obra, unos $2.000 \mathrm{~m}^{2}$ y del volumen de hormigón $\mathrm{H}-375$ del tablero, $1.144 \mathrm{~m}^{3}$, pensamos en dividir, su construcción en fases (Figs. 8 y 9), organizadas de forma que se limitase cada una a menos de $500 \mathrm{~m}^{3}$ de hormigón, tanto por problemas de plazo de hormigonado como por los eventuales de suministro.

Todo el encofrado del tablero se situó sobre cimbra.
La primera fase se configuró con los 4 primeros vanos de zona recta, a partir del estribo 2 , incluyendo la zona común con la corona y sendos vanos curvos, de 20,88 m, medidos en el eje. Los extremos de esta fase los forman voladizos de aproximadamente $1 / 5$ de la luz adyacente, tanto en la zona recta, de 5,20 m de luz, como en la curva, de 4,50 m.

La segunda fase abarcó, en la zona recta el resto del vano de $26 \mathrm{~m}$ y el adyacente al estribo 1 , de $18 \mathrm{~m}$, con una longitud total de $39,30 \mathrm{~m}$.

La fase 3 completaba la corona circular y el tramo trapecial cercano al estribo 3 .

La solución de los cimientos consistió en zapatas convencionales en las pilas, salvo en la pila 1, bajo el tramo recto, que se alza sobre un sólo pilote de $1,50 \mathrm{~m}$ de diámetro. El apoyo en los estribos 1 y 2 , que no soportan empujes de tierras, confiados a muros independientes, se cimentan en dos pilotes de $10 \mathrm{~m}$ de longitud y en unos dados de $4 \mathrm{~m}$ de altura, respectivamente.

El estribo 3 se conforma mediante un gran cabecero, que reposa sobre sendos pilotes de $1,50 \mathrm{~m}$ de diámetro, perforados desde el terraplén ya construido.

En el pretensado (Figs. 10, 11, 12 y 13) del tablero se dispusieron los anclajes solapando cordones, para evitar que un eventual tapón en las vainas provocase una situación incontrolada. Se emplearon cordones $19 \mathrm{~T} 15 \mathrm{y}$, dado el número de las vainas y el poco canto del núcleo, fue muy problemático armonizar sus trazados, creando ventanas de tesado de grandes dimensiones. Este problema se maximizo en la fase 3, dado que la única superficie franca era la frontal del estribo 3, teniendo que enmarcar con cables la zona interior de la corona para garantizar un reparto de tensiones adecuado, lo que obligó a sacarlos $2 / 3$ en la junta de las fases y en la zona trapecial.

Aún así, no fue materialmente posible evitar que en la junta antes aludida dejásemos un par de cordones pasantes con anclajes ciegos, sin ninguna consecuencia posterior.

En cualquier caso, dada la forma de la obra, no hubiera sido nada fácil la solución con conectores.

La salida de los cordones en el estribo 3, correspondiente a la fase 3, se hizo manteniendo el trazado en planta lo más suave posible, lo que provocó una disposición divergente que obligó a tesar la riostra transversalmente para evitar una tracción en la zona.

Todo este conjunto de heterogeneidades hacían que el tablero fuese muy complejo de construir, reto que el personal de obra aceptó con gran deportividad. 


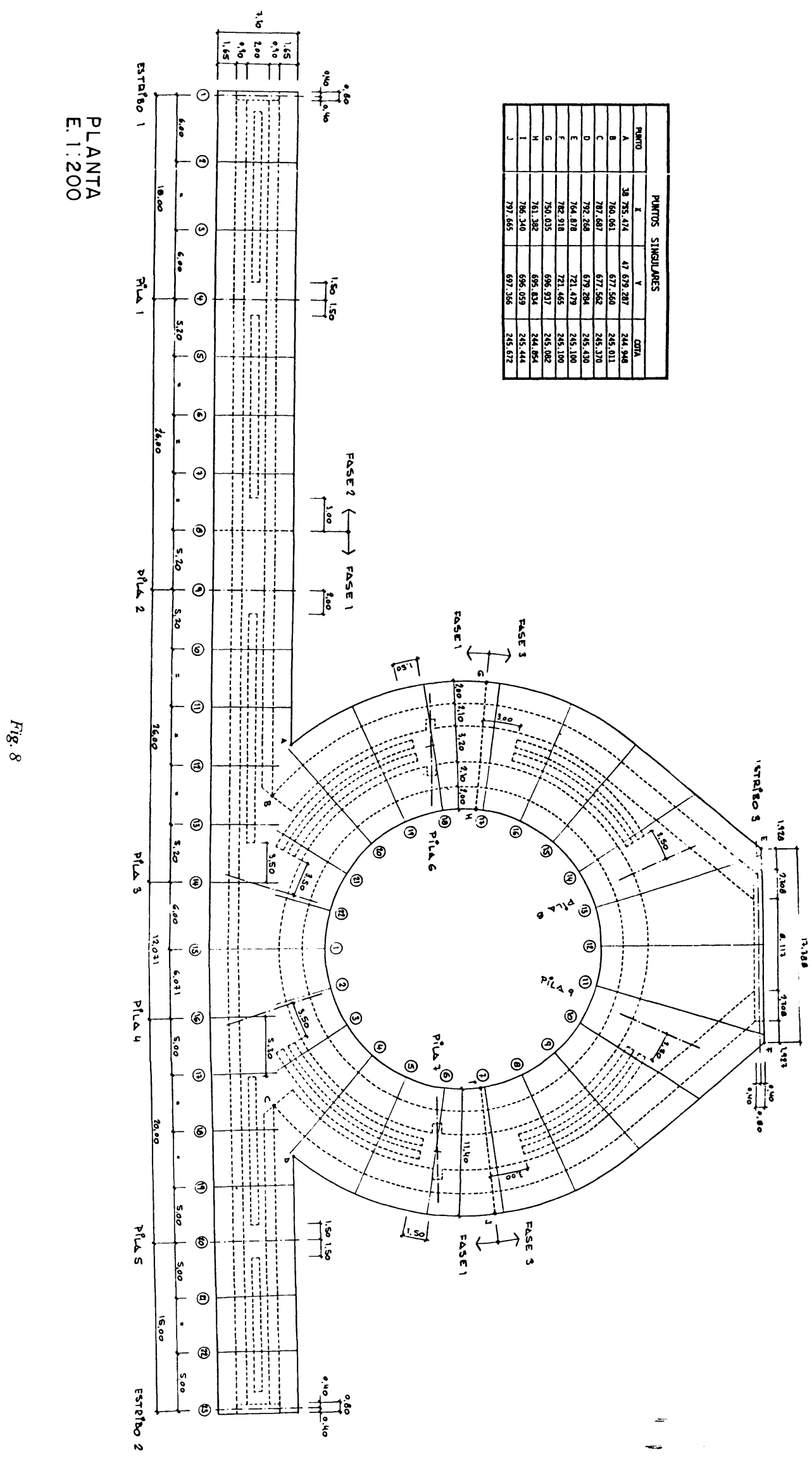




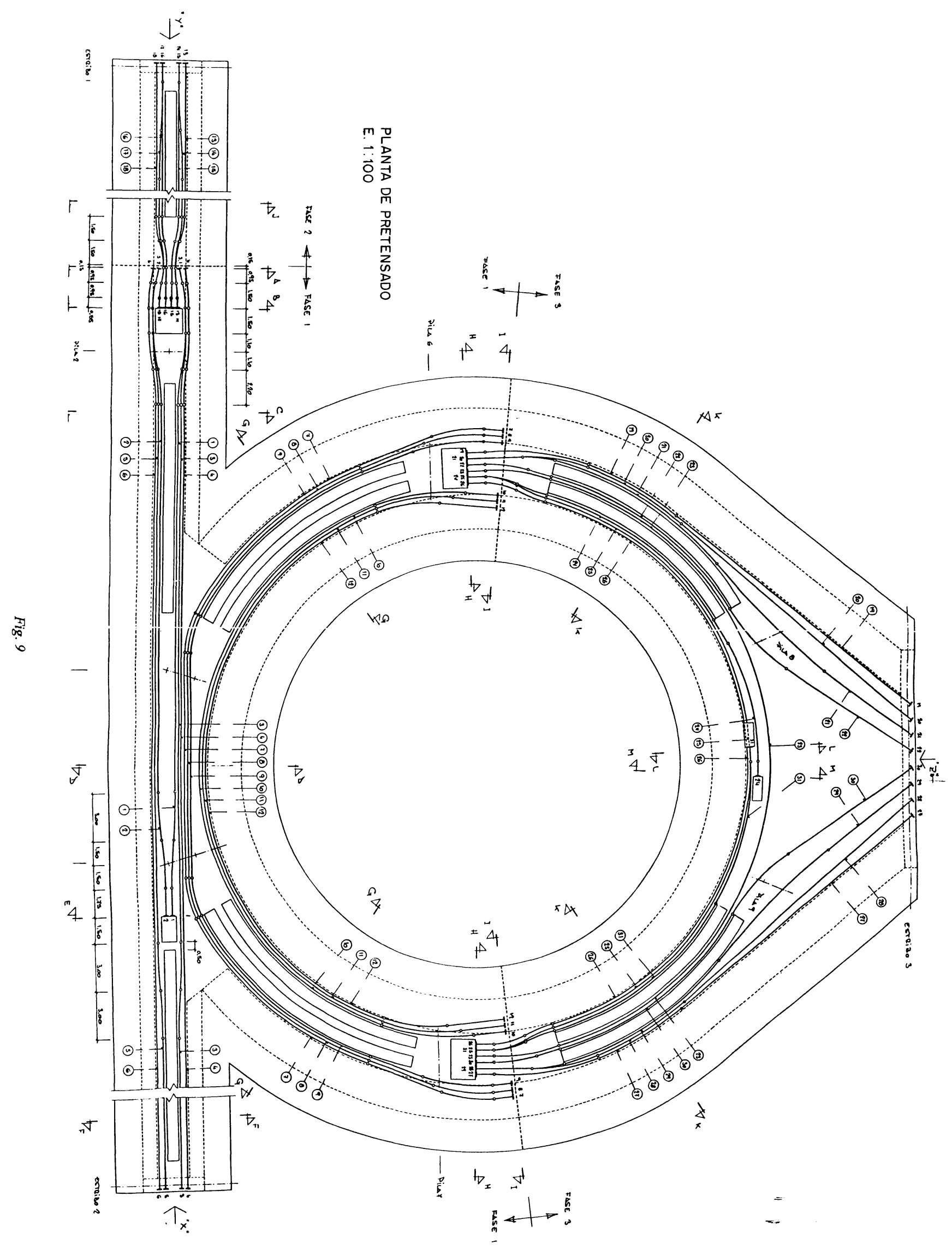




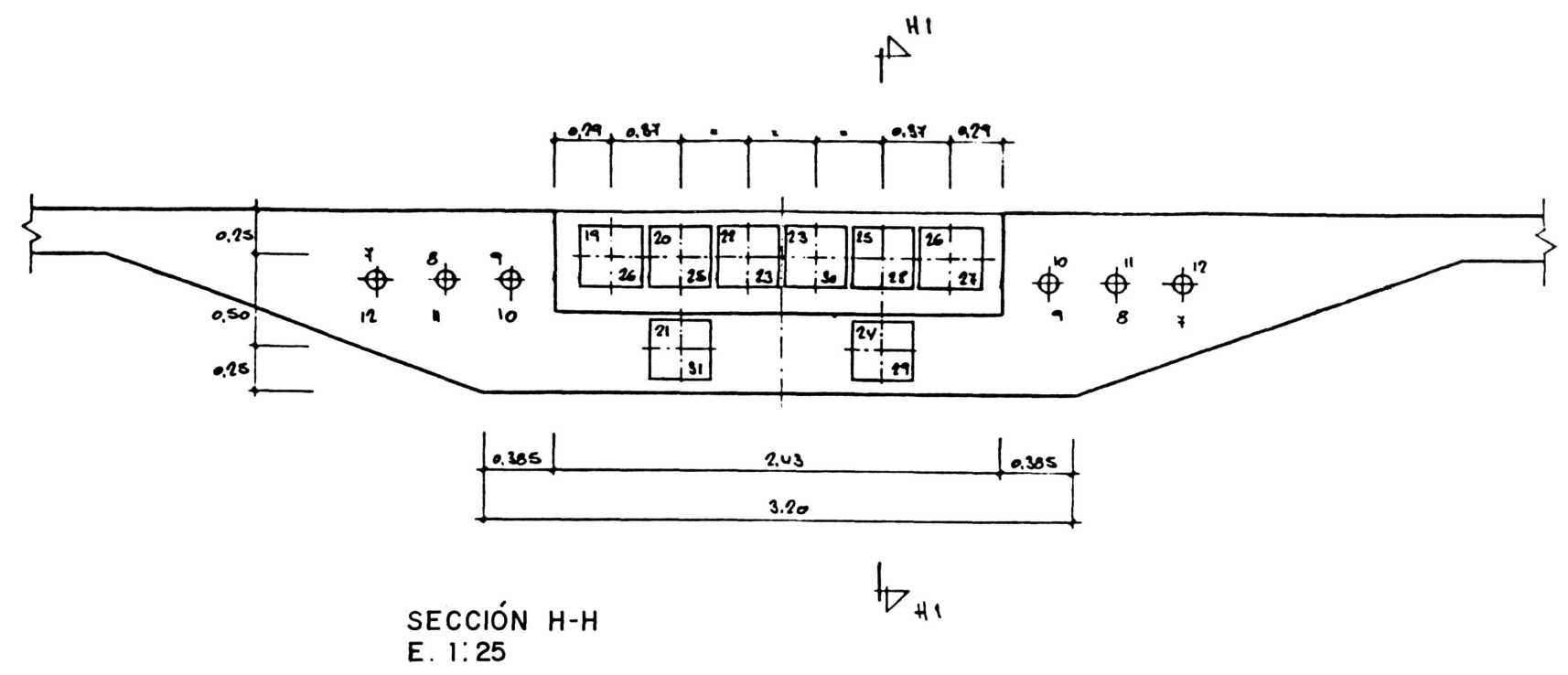

Fig. 10

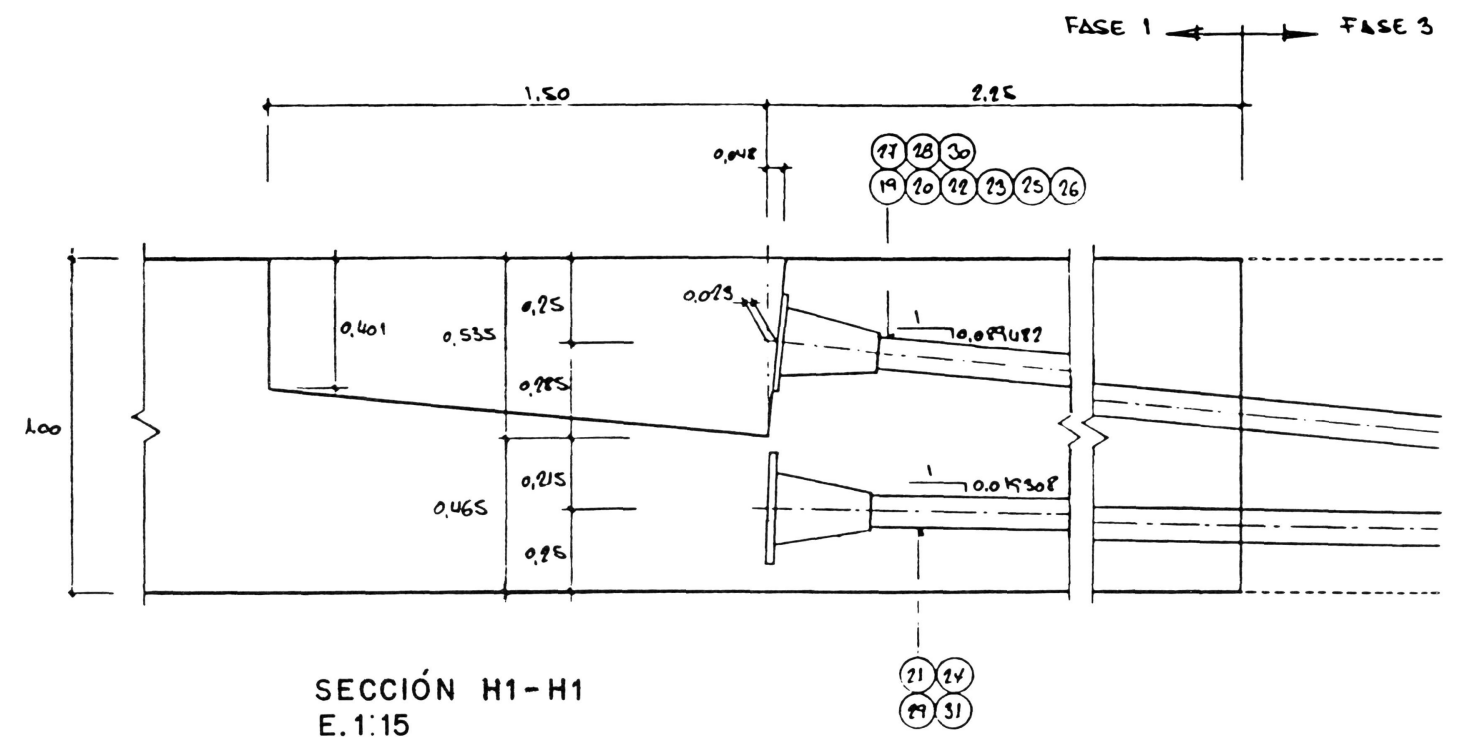

Fig. 11
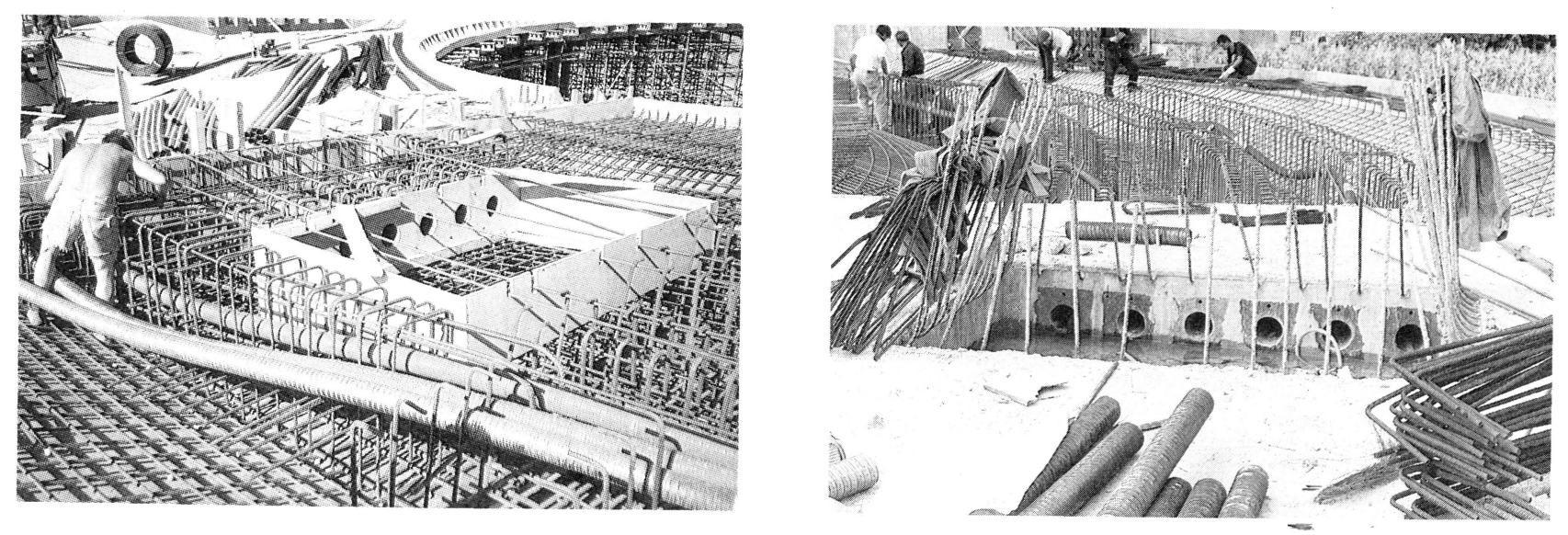

Fotos 3 y 4.- Detalles del pretensado. 

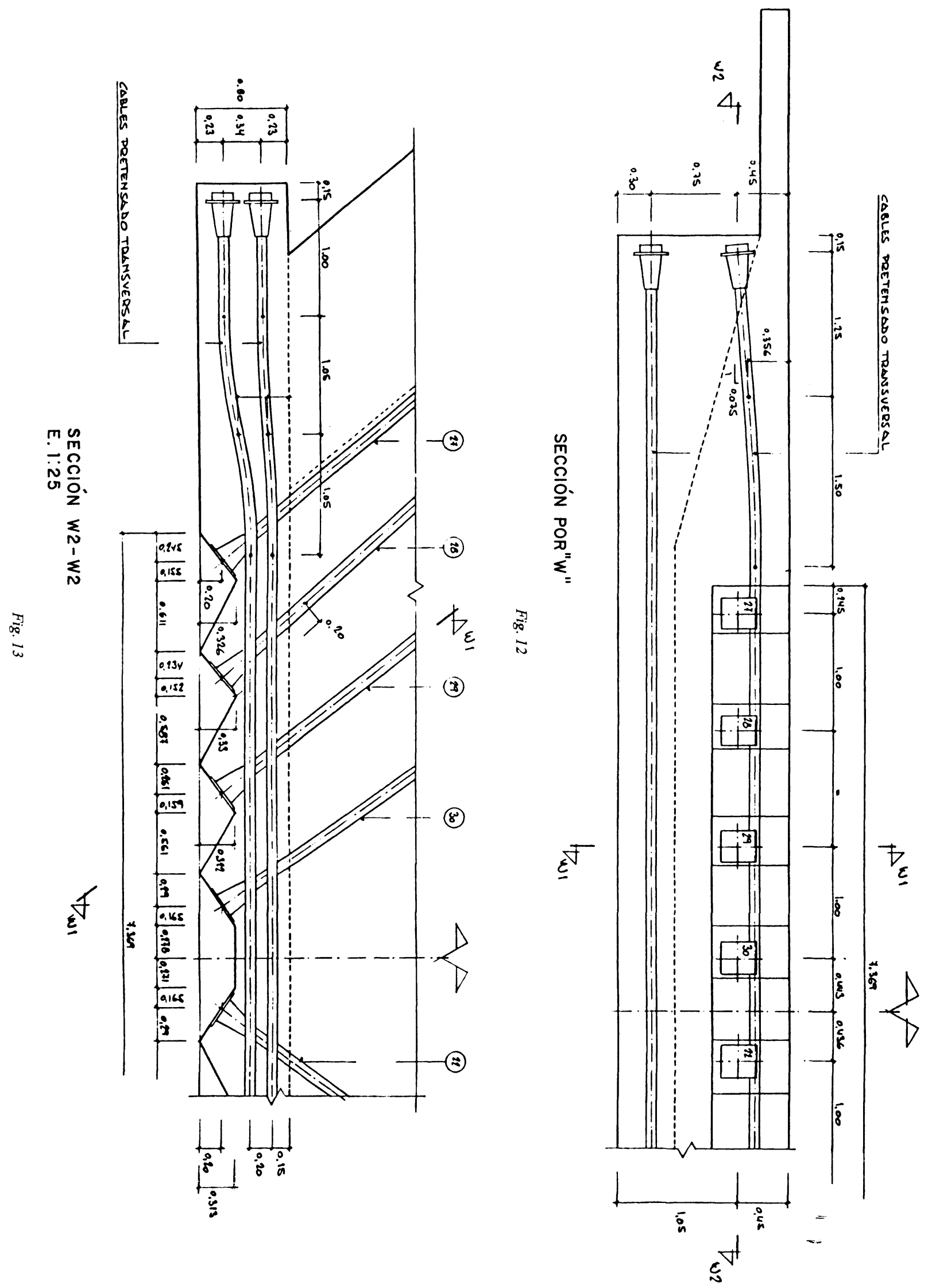


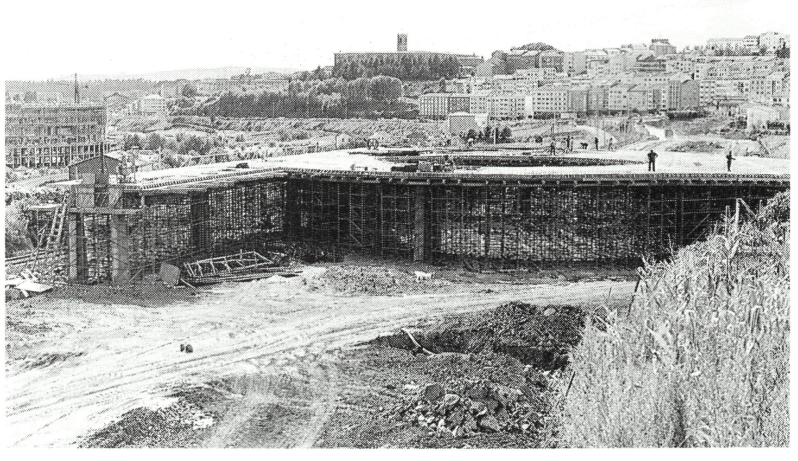

Foto 5

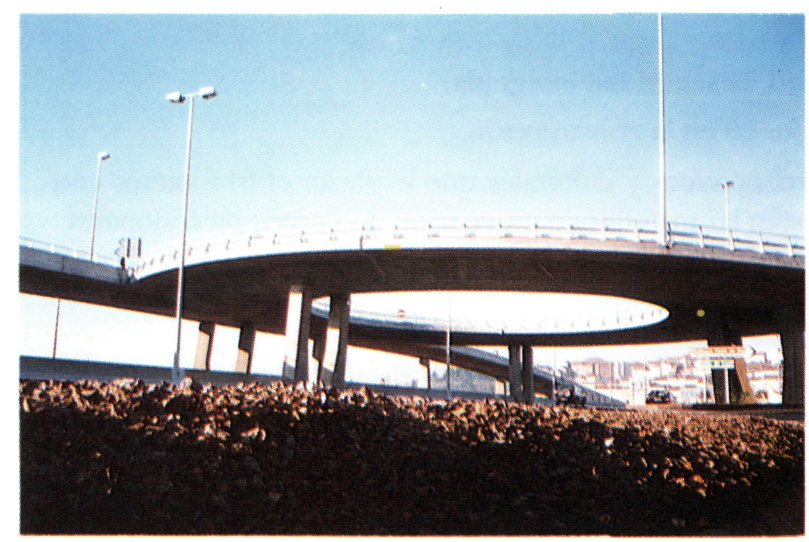

Foto 6

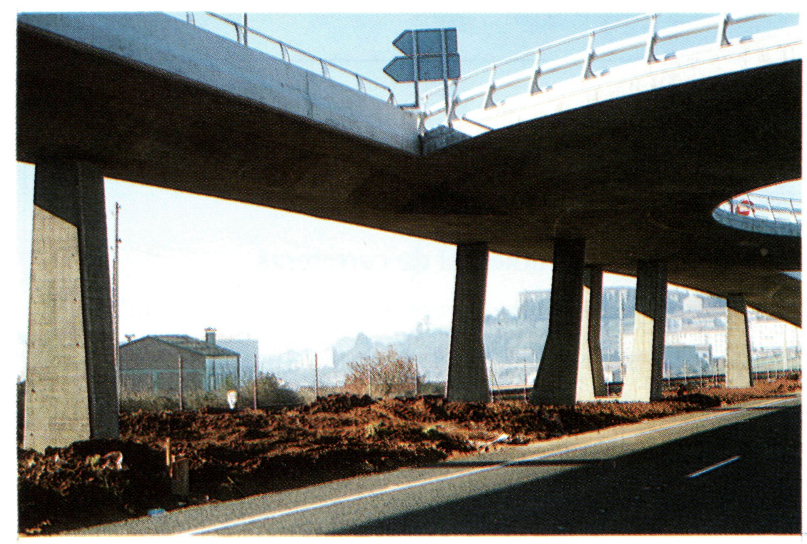

Foto 7

\section{Cálculo}

El cálculo de esfuerzos en el tablero se organizó medianteun estudio de su distribución, en el sentido del ancho, mediante la idealización de zonas localizadas a un emparrillado plano, usando barras de directriz curva.

De este modo se obtenían, para cada caso:

- Distribución de esfuerzos longitudinales.

- Relación torsión-flexión transversal.

- Efecto de diferencia de luces externa/interna, en los tramos curvos

Posteriormente, en un emparrillado global, para cada fase, se obtuvieron los esfuerzos a todo lo ancho.

En todos los emparrillados se tomó una relación $\mathrm{G} / \mathrm{E}=1 / 3$.

Se diseñó el tramo recto en Clase II, y la zona curva en Clase I.

Los efectos de la fluencia, debidos a la evolución del sistema estático, se obtuvieron superponiendo los esfuerzos de fase, multiplicados por 0,70 , a los obtenidos en la estructura completa, multiplicados por 0,30. En cualquier caso resultaron ser prácticamente despreciables.

\section{Materiales empleados}

Las cuantías resultantes en el tablero fueron:

- Hormigón H-375 0,57 $\mathrm{m}^{3} / \mathrm{m}^{2}$

- Acero AEH $500110 \mathrm{~kg} / \mathrm{m}^{3}$

- Acero de pretensar $28 \mathrm{~kg} / \mathrm{m}^{3}$

\section{Equipo de obra}

El Director de la obra, propiedad del M.O.P.T.M.A. fue D. Angel González del Río y el Jefe de obra, por parte de Ferrovial, D. Manuel Berdeal, compañeros de una categoría técnica y humana que invita a volver a compartir una obra con ellos. 